\title{
ON LOMMEL AND BESSEL POLYNOMIALS
}

\section{DAVID DICKINSON ${ }^{1}$}

1. Introduction. The Lommel "polynomials" $R_{n, \nu}(x)$ arise in the theory of Bessel functions. See Watson $[10, \S 9.6-9.73] .^{2}$ If we define the modified Lommel polynomials $h_{n, \nu}(x)$ by

$$
h_{n, \nu}(x)=R_{n, \nu}(1 / x),
$$

we have a polynomial set that obeys

$$
h_{n, \nu}(x)=2 x(n+\nu-1) h_{n-1, \nu}(x)-h_{n-2, \nu}(x) .
$$

It was noted by Hahn [5] that this is an orthogonal type recurrence relation. In this paper, we shall establish explicitly the orthogonality of the $h_{n, v}(x)$ and will present some properties of the Bessel polynomials $y_{n}(x)$, a polynomial set that is orthogonal in a special sense and whose theory in many ways parallels that of the modified Lommel polynomials.

From some formulas involving Lommel polynomials (see Watson $[10, \S 9.6])$, we may define the modified Lommel polynomials $h_{n, \nu}(x)$ in terms of Bessel functions by

$$
J_{\nu+n}(1 / x)=J_{\nu}(1 / x) h_{n, \nu}(x)-J_{\nu-1}(1 / x) h_{n-1, \nu+1}(x),
$$

where $n$ is an integer and $\nu$ is not a nonpositive integer, or by

$$
\begin{aligned}
\pi^{-1}(2 x \sin \pi \nu) & h_{n, \nu}(x) \\
& =J_{\nu+n}(1 / x) J_{-\nu+1}(1 / x)+(-1)^{n} J_{-\nu-n}(1 / x) J_{\nu-1}(1 / x),
\end{aligned}
$$

for $n$ integral and $\nu$ not an even integer. The polynomials have the hypergeometric representation (for $n>1$ )

$h_{n, \nu}(x)=(\nu)_{n}(2 x)^{n}{ }_{2} F_{3}\left(-n / 2,(-n+1) / 2 ; \nu,-n, 1-\nu-n ;-1 / x^{2}\right)$, where

$$
(\nu)_{n}=\frac{\Gamma(\nu+n)}{\Gamma(\nu)} .
$$

Explicitly, the first few polynomials are

Presented to the Society, October 24, 1953; received by the editors April 2, 1954.

1 This paper is part of the author's doctoral dissertation submitted to the University of Michigan, 1953. Portions of this paper were completed while the author was under contract to the Office of Naval Research.

2 Numbers in brackets refer to the references at the end of the paper. 


$$
\begin{aligned}
h_{-1, \nu}(x) & =0, \\
h_{0, \nu}(x) & =1, \\
h_{1, \nu}(x) & =2 \nu x, \\
h_{2, \nu}(x) & =-1+(\nu)(\nu+1) 2^{2} x^{2}, \\
h_{3, \nu}(x) & =-2(\nu+1) 2 x+(\nu)(\nu+1)(\nu+2) 2^{3} x^{3} .
\end{aligned}
$$

2. The orthogonality of the $h_{n, \nu}(x)$. In this section we shall always take $n$ to be a non-negative integer and $\nu$ to be a positive number.

If we multiply both sides of (1) by $x^{s} / J_{\nu-1}(1 / x)$ where $s$ is a nonnegative integer, we get

$$
\frac{x^{s} J_{\nu+n}(1 / x)}{J_{\nu-1}(1 / x)}=x^{s} h_{n, \nu}(x) \frac{J_{\nu}(1 / x)}{J_{\nu-1}(1 / x)}-x^{s} h_{n-1, \nu+1}(x) .
$$

Now the two terms involving the Bessel function quotients are analytic outside any circle that contains the finite zeros of $J_{\nu-1}(1 / x)$. Hence each possesses a Laurent expansion about the origin that converges uniformly on and in any annulus whose inside boundary has the finite zeros of $J_{\nu-1}(1 / x)$ in its interior. If we integrate these expansions around a contour $C_{\nu}$ that encircles the origin in a positive direction and that lies within the annulus, all of the terms vanish except, if such exists, the term involving $1 / x$. Since

$$
\frac{x^{s} J_{\nu+n}(1 / x)}{J_{\nu-1}(1 / x)}=\frac{x^{s-n-1}}{2^{n+1}(\nu)_{n+1}}\left\{1+\frac{(n+1)}{4(\nu)(\nu+n+1) x^{2}}+\cdots\right\},
$$

the integral around $C_{\nu}$ of the left member of (3) vanishes for $(s-n)$ $<0$. Since the second member of the right side of (3) is a polynomial, its integral around $C_{\nu}$ vanishes. Thus we are left with

$$
\frac{1}{2 \pi i} \int_{C_{\nu}} x^{s} h_{n, \nu}(x) \frac{J_{\nu}(1 / x)}{J_{\nu-1}(1 / x)} d x=\left\{\begin{array}{cl}
0 & \text { for } s<n, \\
\frac{1}{2^{n+1}(\nu)_{n+1}} & \text { for } s=n .
\end{array}\right.
$$

If we set $n=0$ in (4) it is not hard to see that for the moments of $h_{n, \nu}(x)$,

$$
m_{s, \nu}=\frac{1}{2 \pi i} \int_{C_{\nu}} x^{s} \frac{J_{\nu}(1 / x)}{J_{\nu-1}(1 / x)} d x
$$

we have

$$
\frac{J_{\nu}(1 / x)}{J_{\nu-1}(1 / x)}=\sum_{s=0}^{\infty} m_{s, \nu} x^{-1-s}
$$


The question naturally arises as to whether the complex integral (5) can be replaced by a real one. To find some necessary properties of such a real integral, let us first investigate the zeros of $h_{n, \nu}(x)$.

That the zeros of $h_{n, v}(x)$ are all real follows from the recurrence relation (see Szegö $[8, \S 3.3,(4)]$ or Sheffer [7, Theorem 3]). that

We have from a theorem of Hurwitz (see Watson $[10, \S 9.65]$ )

$$
\lim _{n \rightarrow \infty} \frac{(2 x)^{1-\nu-n} h_{n, \nu}(x)}{\Gamma(n+\nu)}=J_{\nu-1}(1 / x)
$$

uniformly for all $x$ in any closed and bounded annular region centered about the origin. This implies, from another theorem of Hurwitz (see Titchmarsh [9, p. 119]), that if $[c, d]$ is any real closed interval not containing any zeros of $J_{\nu-1}(1 / x)$, then for $n$ sufficiently large, $h_{n, \nu}(x)$ has no zeros in $[c, d]$. But this implies (see Szegö $[8$, Theorem 6.1.1]) that if the $h_{n, \nu}(x)$ are orthogonal over a real interval $[a, b]$ with respect to a nondecreasing distribution $d \alpha_{\nu}(x)$, the integral of $d \alpha_{\nu}(x)$ over any subinterval, such as the interval $[c, d]$, vanishes. Hence it is necessary that $d \alpha_{\nu}(x)$ be a step function with points of increase at the zeros of $J_{\nu-1}(1 / x)$.

To summarize, we have that if the $h_{n, \nu}(x)$ are orthogonal with respect to a nondecreasing distribution $d \alpha_{\nu}(x)$, we must have

$$
m_{s, \nu}=\int x^{s} d \alpha_{\nu}(x)=\sum_{n} I_{n} p_{n}^{s}
$$

where the distribution $d \alpha_{\nu}(x)$ has an increase of $I_{n}$ at the point $p_{n}$ and where the integration and summation are taken over appropriate domains.

When $\nu=1 / 2$, the quotient of Bessel functions appearing in (6) becomes $\tan 1 / x$ and we have

$$
\tan 1 / x=\sum_{i=0}^{\infty} m_{2 i, 1 / 2} x^{-1-2 i}=\sum_{i=0}^{\infty} \frac{4^{i+1}\left(4^{i+1}-1\right) B_{i+1}}{(2 i+2) !} x^{-1-2 i}
$$

where the $B_{i}$ are the Bernoulli numbers. The coefficients in (9) may be expressed in terms of the generalized zeta function (see [11, $\S 13.151$, Ex. $1 ; \S 13.13$, Ex. 2$])$. Thus we have that

$$
m_{s, 1 / 2}=\frac{2}{\pi^{s+2}} \zeta(s+2,1 / 2)=2 \sum_{n=0}^{\infty} \frac{1}{[(n+1 / 2) \pi]^{s+2}}
$$

provided $s$ is even. Now (10) is not true when $s$ is odd since the odd moments vanish. We consider the expression 


$$
m_{s, 1 / 2}=\sum_{n=-\infty}^{+\infty} \frac{1}{[(n+1 / 2) \pi]^{s}[(n+1 / 2) \pi]^{2}} .
$$

When $s$ is even, the summation in (11) reduces to that of (10) and, when $s$ is odd, the sum vanishes. But (11) is a sum of the form of (8). Hence we have that

$$
\int_{-2 / \pi}^{+2 / \pi} h_{n, 1 / 2}(x) h_{m, 1 / 2}(x) d \alpha_{1 / 2}(x)=\left\{\begin{array}{cl}
0 & \text { for } m \neq n, \\
\frac{2^{n} n !}{(2 n+1) !} & \text { for } m=n,
\end{array}\right.
$$

where $d \alpha_{1 / 2}(x)$ is a nondecreasing step function having an increase of $[(n+1 / 2) \pi]^{-2}$ at the point $x=[(n+) 1 / 2 \pi]^{-1}$ for $n=0, \pm 1, \pm 2, \cdots$.

Using as a guide this last paragraph, we may proceed to a real integral representation of the moments of the general $h_{n, \nu}(x)$.

Let all of the nonvanishing zeros $j_{\nu, n}$ of $J_{\nu}(x)$ have the order they have on the real line and let, in particular, $j_{\nu, 1}$ be the smallest positive one. From the well known recurrence relation for Bessel functions

$$
x J_{\nu-1}^{\prime}(x)=(\nu-1) J_{\nu-1}(x)-x J_{\nu}(x)
$$

we may write, provided $0<x<j_{\nu-1,1}$,

$$
\frac{J_{\nu-1}^{\prime}(x)}{J_{\nu-1}(x)}=\frac{d}{d x} \ln J_{\nu-1}(x)=\frac{(\nu-1)}{x}-\frac{J_{\nu}(x)}{J_{\nu-1}(x)} .
$$

From Watson $[10, \S 15.41]$, we have

$$
J_{\nu-1}(x)=\frac{(x / 2)^{\nu-1}}{\Gamma(\nu)} \prod_{n=1}^{\infty}\left(1-\frac{x^{2}}{j_{\nu-1, n}}\right)
$$

and hence, again provided $0<x<j_{\nu-1,1}$, we have the uniformly convergent series of analytic functions

$$
\ln J_{\nu-1}(x)=\ln \frac{(x / 2)^{\nu-1}}{\Gamma(\nu)}+\sum_{n=1}^{\infty} \ln \left(1-\frac{x^{2}}{j_{\nu-1, n}}\right) .
$$

Taking the derivative of both sides of (14), we have

$$
\frac{d}{d x} \ln J_{\nu-1}(x)=\frac{\nu-1}{x}+\sum_{n=1}^{\infty}\left(\frac{-2 x}{j_{\nu-1, n}^{2}}\right)\left(1-\frac{x^{2}}{j_{\nu-1, n}^{2}}\right)^{-1}
$$

and hence

$$
\frac{d}{d x} \ln J_{\nu-1}(x)=\frac{\nu-1}{x}-\sum_{n=1}^{\infty} \sum_{k=0}^{\infty} \frac{2 x^{2 k+1}}{j_{\nu-1, n}^{2 k+2}}
$$


Now all the summands in the convergent double series are positive and we may interchange the order of summation. If we interchange the summations and compare the resulting expression with (13), we see that

$$
\frac{J_{\nu}(x)}{J_{\nu-1}(x)}=\sum_{k=0}^{\infty}\left(2 \sum_{n=1}^{\infty} \frac{1}{j_{\nu-1, n}^{2 k+2}}\right) x^{2 k+1}
$$

or, from (6),

$$
m_{s, \nu}=2 \sum_{n=1}^{\infty} \frac{1}{j_{\nu-1, n}^{2 s+2}}
$$

for $s$ even. To find an expression for the $m_{s, \nu}$ valid for all $s$, we use the reasoning that led from (10) to (11). This leads to the expression

$$
m_{s, \nu}=\sum_{n=-\infty}^{+\infty} j_{v-1, n}^{-8-2}
$$

valid for all $s$. Hence we have

$$
\int_{j^{-1}{ }_{\nu-1,0}^{j-1}{ }_{\nu-1,1}} h_{n, \nu}(x) h_{m, \nu}(x) d \alpha_{\nu}(x)=\left\{\begin{array}{cl}
0 & \text { for } m \neq n, \\
\frac{1}{2^{n+1}(\nu)_{n+1}} & \text { for } m=n,
\end{array}\right.
$$

where $d \alpha_{\nu}(x)$ is a nondecreasing step function having an increase of $j_{\nu-1, n}^{-2}$ at the point $x=j_{\nu-1, n}^{-1}$ for $n=0, \pm 1, \pm 2, \cdots$.

Approaching the problem from a different angle, H. O. Pollak and G. H. Wannier have independently established the integral (15).

3. Some relations between the Bessel polynomials and the modified Lommel polynomials. The Bessel polynomials $y_{n}(x)$ may be defind by the relation (see [6])

$$
J_{n+1 / 2}(1 / x)=(x / 2 \pi)^{1 / 2}\left[i^{-n-1} e^{i / x} y_{n}(i x)+i^{n+1} e^{-i / x} y_{n}(-i x)\right]
$$

for $n$ integral. They have the hypergeometric representation

$$
y_{n}(x)={ }_{2} F_{0}(-n, n+1 ;-;-x / 2)
$$

and hence

$$
y_{n}(x)=y_{-n-1}(x) .
$$

If we use (16) to express the right member of (2) in terms of exponentials and Bessel polynomials, the exponentials drop out and we have

$$
h_{n, 8+1 / 2}(x)=2^{-1}\left[i^{-n} y_{n+s}(i x) y_{s-1}(-i x)+i^{n} y_{n+s}(-i x) y_{8-1}(i x)\right]
$$


for $s$ integral. An interesting special case of (17) is

$$
h_{n, 1 / 2}(x)=2^{-1}\left[i^{-n} y_{n}(i x)+i^{n} y_{n}(-i x)\right] \text {. }
$$

Now (17) may be used to find an expression for the product of two Bessel polynomials in terms of a sum of two modified Lommel polynomials. In (17), we shift the parameter $s$ to $s-1$ and the index $n$ to $n+2 s-1$ :

$$
\begin{aligned}
h_{n+2 s-1,-s+3 / 2}(x)=2^{-1}\left[i^{-n-2 s+1} y_{n+8}\right. & (i x) y_{-8}(-i x) \\
& \left.+i^{n+2 s-1} y_{n+s}(-i x) y_{-s}(i x)\right] .
\end{aligned}
$$

Since

$$
y_{-8}(x)=y_{s-1}(x),
$$

we may eliminate one of the Bessel polynomial products between (17) and (19). This gives us

$$
h_{n, s+1 / 2}(x)+i^{2 s+1} h_{n+2 s-1,-s+3 / 2}(x)=i^{n} y_{n+s}(-i x) y_{s-1}(i x) .
$$

4. Some generating functions. We may use the known generating functions for the $y_{n}(x)$ to form some new generating functions for the $h_{n, 1 / 2}(x)$. For example, from [6] we have the divergent generating function for the $y_{n}(x)$ :

$$
\frac{1}{1-t}{ }_{2} F_{0}\left(1,1 / 2 ;-; \frac{-2 x t}{(1-t)^{2}}\right) \sim \sum_{n=0}^{\infty} y_{n}(x) t^{n} .
$$

From this generating function, we may form a generating function for the right member of (18). It follows that

$$
\begin{aligned}
& \frac{1}{1-i t}{ }_{2} F_{0}\left(1,1 / 2 ;-; \frac{2 x t}{(1-i t)^{2}}\right) \\
& \quad+\frac{1}{1+i t}{ }_{2} F_{0}\left(1,1 / 2 ;-; \frac{2 x t}{(1+i t)^{2}}\right) \sim 2 \sum_{n=0}^{\infty} h_{n, 1 / 2}(x) t^{n} .
\end{aligned}
$$

Similarly, we may use (16) to form some apparently new generating functions for $J_{n+1 / 2}(x)$. For example, from the divergent generating function (see [1]),

$$
\begin{aligned}
& { }_{2} F_{0}\left(\alpha, 1-\alpha ;-; \frac{t-\left(t^{2}+2 x t\right)^{1 / 2}}{2}\right) \\
& \quad{ }_{2} F_{0}\left(\alpha, 1-\alpha ;-; \frac{t+\left(t^{2}+2 x t\right)^{1 / 2}}{2}\right) \sim \sum_{n=0}^{\infty} \frac{(\alpha)_{n}(1-\alpha)_{n}}{n !} y_{n}(x) t^{n}
\end{aligned}
$$

where $\alpha$ is an arbitrary parameter, we may write 


$$
\begin{array}{r}
-i(2 \pi x)^{-1 / 2} e^{i x}{ }_{2} F_{0}\left(\alpha, 1-\alpha ;-; \frac{-i t-\left(-t^{2}+2 t / x\right)^{1 / 2}}{2}\right) \\
\cdot{ }_{2} F_{0}\left(\alpha, 1-\alpha ;-; \frac{-i t+\left(-t^{2}+2 t / x\right)^{1 / 2}}{2}\right) \\
+i(2 \pi x)^{-1 / 2} e^{-i x} F_{0}\left(\alpha, 1-\alpha ;-; \frac{i t-\left(-t^{2}+2 t / x\right)^{1 / 2}}{2}\right) \\
\cdot{ }_{2} F_{0}\left(\alpha, 1-\alpha ;-; \frac{i t+\left(-t^{2}+2 t / x\right)^{1 / 2}}{2}\right) \\
\sim \sum_{n=0}^{\infty} \frac{(\alpha)_{n}(1-\alpha)_{n}}{n !} J_{n+1 / 2}(x) t^{n} .
\end{array}
$$

When $\alpha$ is a negative integer $-p,(22)$ is no longer divergent and is a finite generating function, the summation stopping at $n=p$.

5. A general theorem on finite sums of Bessel polynomials. Following a technique that Nielsen used in working with Lommel polynomials (see $[10, \S 9.62]$ ), we have the

THEOREM. If

$$
\sum_{n \in D} f_{n}(x) J_{n+8+1 / 2}(x) \equiv 0
$$

where the $f_{n}(x)$ are algebraic functions of $s$, where $s$ is an integer, and where the sum is taken over $D$, a finite set of integers, then it follows that

$$
\sum_{n \in D} f_{n}(1 / i x) i^{n} y_{n+\varepsilon}(x) \equiv 0
$$

and

$$
\sum_{n \in D} f_{n}(-1 / i x) i^{-n} y_{n+s}(x) \equiv 0 .
$$

Proof. From (16), (23) may be written

$$
\sum_{n \in D} f_{n}(x)(2 \pi x)^{-1 / 2}\left[i^{-n-s-1} e^{i x} y_{n+s}(-1 / i x)+i^{n+8+1} e^{-i x} y_{n+s}(1 / i x)\right] \equiv 0
$$

or

$$
e^{2 i x} \sum_{n \in D} f_{n}(x) i^{-n-s} y_{n+s}(-1 / i x)-\sum_{n \in D} f_{n}(x) i^{n+s} y_{n+s}(1 / i x) \equiv 0 .
$$

But (26) is an expression of the form $A B-C \equiv 0$ where $A$ is transcendental and $B$ and $C$ are algebraic. Hence $B$ and $C$ are zero.

For example, from an algebraic relation between any three Bessel 
functions whose orders differ by integers $[10, \S 9.64]$ we may write

$$
\begin{aligned}
& J_{n+1 / 2}(x) h_{p-m-1, m+3 / 2}(1 / x) \\
& +J_{m+1 / 2}(x) h_{n-p-1, p+3 / 2}(1 / x) \\
& +J_{p+1 / 2}(x) h_{m-n-1, n+3 / 2}(1 / x)=0 .
\end{aligned}
$$

From this it follows that

$$
\begin{aligned}
& i^{n} y_{n}(x) h_{p-m-1, m+3 / 2}(i x) \\
& +i^{m} y_{m}(x) h_{n-p-1, p+3 / 2}(i x) \\
& +i^{p} y_{p}(x) h_{m-n-1, n+3 / 2}(i x)=0 .
\end{aligned}
$$

Also, setting $\mu=s+1 / 2$ in Watson $[10, \S 5.21$, (2)], we have

$$
(x / 2)^{-n} J_{n+8+1 / 2}(x)=\sum_{k=0}^{n}\left(\begin{array}{l}
n \\
k
\end{array}\right) \frac{(2 k+s+1 / 2)}{(s+k+1 / 2)_{n+1}} J_{s+1 / 2+2 k}(x)
$$

and hence

$$
(-2 x)^{n} y_{n+s}(x)=\sum_{k=0}^{n}\left(\begin{array}{l}
n \\
k
\end{array}\right) \frac{(2 k+s+1 / 2)}{(s+k+1 / 2)_{n+1}} y_{s+2 k}(x) .
$$

Invoking the relation $y_{n}(x)=y_{-n-1}(x)$, we have, after some algebraic manipulation of (28), a relation involving the $y_{n}(x)$ with non-negative indices only:

$$
x^{n}=\sum_{k=0}^{n} \frac{n !(2 n-2 k+1) 2^{n}(-1)^{k}}{(2 n-k+1) ! k !} y_{n-k}(x) .
$$

Expression (29) may be used to express formally in terms of Bessel functions any function given as a power series.

6. Concerning the zeros of the Bessel polynomials. If we set $n=0$ in (20), we have

$$
1+i^{2 s+1} h_{2 s-1,-s+3 / 2}(x)=y_{s}(-i x) y_{s-1}(i x) .
$$

Since, for $\nu$ or $s$ real, all the Lommel polynomials have real coefficients, the left member and hence also the right member of (30) have no real zeros. Therefore no Bessel polynomial $y_{n}(x)$ has a purely imaginary zero.

We may now show that no two Bessel polynomials have a zero in common. For suppose there were a number $\alpha$ such that $y_{n}(\alpha)=y_{m}(\alpha)$ $=0$ where $m>n>0$. Then, by (27),

$$
i^{p} y_{p}(\alpha) h_{m-n-1, n+3 / 2}(i \alpha)_{;}^{j}={ }_{-} 0
$$


for an arbitrary integer $p$. Now $h_{m-n-1, n+3 / 2}(i x)$, having a nonnegative index and a positive parameter, has, from $\$ 2$, only purely imaginary zeros. But from the preceding paragraph, $y_{n}(x)$ has no purely imaginary zeros. Thus $y_{p}(\alpha)=0$ or, in particular, $y_{0}(\alpha)=1=0$ which is absurd.

If, in (20), we set $s=0$, we obtain the relation

$$
h_{n, 1 / 2}(x)+i h_{n-1,3 / 2}(x)=i^{n} y_{n}(-i x) .
$$

Hence, from this and (7), we have Grosswald's limit [4]:

$$
\lim _{n \rightarrow \infty} \frac{\pi^{1 / 2}(2 x)^{-n} y_{n}(x)}{\Gamma(n+1 / 2)}=e^{1 / x}
$$

uniformly in any closed and bounded annular region $A$ centered about the origin. From a theorem of Hurwitz (see Titchmarsh [9, p. 119]) it follows that for any such region $A$, the zeros of $y_{n}(x)$, for a sufficiently large $n$, will not lie in $A$. Since the zeros of $y_{n}(x)$ are bounded (see [4]), it follows that for any $\epsilon>0$, the zeros of $y_{n}(x)$, for sufficiently large $n$, lie within $|x|=\epsilon$.

7. A characterization of orthogonality. If we set $n=0$ in (17), we obtain after some simplification

$$
2=y_{8}(x) y_{8-1}(-x)+y_{8}(-x) y_{8-1}(x) .
$$

This last expression is almost enough to imply the existence of an orthogonal type recurrence relation. Specifically, we have the theorem and corollary:

Theorem. For a set of polynomials $p_{n}(x), n=0,1, \cdots$, where each $p_{n}(x)$ is of degree precisely $n$, to have the property

$$
a_{n}=p_{n-1}(x) p_{n}(-x)+p_{n-1}(-x) p_{n}(x)
$$

for some $a_{n} \neq 0, n=1,2, \cdots$, it is necessary and sufficient that there exist a recurrence relation of the form

$$
p_{n}(x)=x B_{n} p_{n-1}(x)+C_{n} p_{n-2}(x), \quad C_{n} \neq 0 \quad(n=2,3, \cdots)
$$

and that $p_{1}(0) \neq 0$.

NeCESSITy PROOF. After setting $x=0$ in (32), it becomes evident that (32) may be written

$$
2 p_{n}(0) p_{n-1}(0)=p_{n-1}(x) p_{n}(-x)+p_{n-1}(-x) p_{n}(x) \quad(n=1,2, \cdots) .
$$

Shifting the index $n$ to $n-1$, we obtain

$$
\begin{array}{r}
2 p_{n-2}(0) p_{n-1}(0)=p_{n-1}(x) p_{n-2}(-x)+p_{n-1}(-x) p_{n-2}(x) \\
(n=2,3, \cdots) .
\end{array}
$$


By Cramer's rule, we may now write

$$
\begin{aligned}
\left|\begin{array}{cc}
p_{n}(-x) & p_{n}(x) \\
p_{n-2}(-x) & p_{n-2}(x)
\end{array}\right| & p_{n-1}(x)=2 p_{n}(0) p_{n-1}(0) p_{n-2}(x) \\
& -2 p_{n-2}(0) p_{n-1}(0) p_{n}(x) \quad(n=2,3, \cdots) .
\end{aligned}
$$

The right member of (34) when evaluated at $x=0$ vanishes. Since $p_{n-1}(0) \neq 0$ (for otherwise we would have $a_{n}=0$ ), it must be the determinant in the left member of (34) that has the factor $x$. Moreover, by comparing the degrees of each member of (34), it is apparent that the determinant is a linear function of $x$. Hence we have, where $f_{n}$ is some nonvanishing function of $n$,

$$
x f_{n} p_{n-1}(x)=2 p_{n}(0) p_{n-1}(0) p_{n-2}(x)-2 p_{n-2}(0) p_{n-1}(0) p_{n}(x)
$$

or

$$
p_{n}(x)=\frac{-x f_{n}}{2 p_{n-2}(0) p_{n-1}(0)} p_{n-1}(x)+\frac{p_{n}(0)}{p_{n-2}(0)} p_{n-2}(x) .
$$

SufficienCy PROOF. From (33), we may write

$$
p_{n}(x) p_{n-1}(-x)=x B_{n} p_{n-1}(x) p_{n-1}(-x)+C_{n} p_{n-2}(x) p_{n-1}(-x)
$$

and also

$$
p_{n}(-x) p_{n-1}(x)=-x B_{n} p_{n-1}(-x) p_{n-1}(x)+C_{n} p_{n-2}(-x) p_{n-1}(x) .
$$

If we add these two equations, we obtain

$$
\begin{aligned}
p_{n}(x) p_{n-1}(-x)+p_{n}(-x) p_{n-1}(x) \\
\quad=C_{n}\left[p_{n-2}(x) p_{n-1}(-x)+p_{n-2}(-x) p_{n-1}(x)\right] .
\end{aligned}
$$

By iterating (35) it is evident that

$p_{n}(x) p_{n-1}(-x)+p_{n}(-x) p_{n-1}(x)$

$$
=\left[p_{1}(x) p_{0}(-x)+p_{1}(-x) p_{0}(x)\right] \prod_{k=2}^{n} C_{k} .
$$

For $n=1$, the right member is $a_{1}=2 p_{1}(0) p_{0}(0)$ and for $n \geqq 2$, it is $a_{n}=2 p_{1}(0) p_{0}(0) \prod_{k=2}^{n} C_{k}$.

Corollary. For a set of polynomials $p_{n}(x), n=0,1, \cdots$, where each $p_{n}(x)$ is of degree precisely $n$, to be orthogonal, it is sufficient that there exist a relation of the form

$$
a_{n}=p_{n}(x) p_{n-1}(x)+p_{n}(-x) p_{n-1}(x)
$$

where $(-1)^{n} a_{1} a_{n}<0$ for $n \geqq 2$. 
Proof. The condition $(-1)^{n} a_{1} a_{n}<0$ implies that $C_{n}<0$ for $n \geqq 2$. The orthogonality then follows from a theorem of Favard [3].

The conditions of this theorem and corollary are met in the specialized Jacobi polynomials $P_{n}^{\alpha,-\alpha}(x)$ where $\alpha^{2}<1$. They have the properties

$$
\begin{aligned}
P_{n}^{(\alpha,-\alpha)}(x)=\frac{x(2 n-1)}{n} P_{n-1}^{(\alpha,-\alpha)}(x)-\frac{(n-1)^{2}-\alpha^{2}}{n(n-1)} P_{n-2}^{(\alpha,-\alpha)}(x), \\
n=2,3, \cdots,
\end{aligned}
$$

and

$$
\begin{array}{r}
\frac{2(-1)^{n}(\alpha)_{n}(-\alpha)_{n}}{\alpha n !(n-1) !}=P_{n}^{(\alpha,-\alpha)}(x) P_{n-1}^{(\alpha,-\alpha)}(-x)+P_{n-1}^{(\alpha,-\alpha)}(x) P_{n}^{(\alpha,-\alpha)}(x), \\
n=1,2, \cdots .
\end{array}
$$

\section{BIBLIOGRAPHY}

1. F. Brafman, $A$ set of generating functions for the Bessel polynomials, Proc. Amer. Math. Soc. vol. 4 (1953) pp. 275-277.

2. Sister M. Celine Fasenmyer, Some generalized hypergeometric polynomials, Bull. Amer. Math. Soc. vol. 53 (1947) pp. 806-812.

3. J. Favard, Sur les polynomes de Tchebicheff, C.R. Acad. Sci. Paris vol. 200 (1935) pp. 2052-2053.

4. E. Grosswald, On some algebraic properties of the Bessel polynomials, Trans. Amer. Math. Soc. vol. 71 (1951) pp. 197-210.

5. W. Hahn, Über orthogonal Polynome mit drei Parametern, Deutsche Math. vol. 5 (1940) pp. 273-278.

6. H. L. Krall and O. Frink, A new class of orthogonal polynomials: The Bessel polynomials, Trans. Amer. Math. Soc. vol. 65 (1949) pp. 100-115.

7. I. M. Sheffer, Note on functionally-orthogonal polynomials, Tohoku Math. J. vol. 33 (1930) pp. 3-11.

8. G. Szegö, Orthogonal polynomials, Amer. Math. Soc. Colloquium Publications, vol. $23,1939$.

9. E. C. Titchmarsh, The theory of functions, London, Oxford University Press, 1939.

10. G. N. Watson, A treatise on the theory of Bessel functions, New York, Macmillan, 1944.

11. E. T. Whittaker and G. N. Watson, A course in modern analysis, New York, Macmillan, 1943.

UNIVERSITY OF MichigAN AND

The Pennsylvania State University 